\title{
Untangling the Complexity in the Galactic Centre: a way to understand the origin of the gamma-ray emission from the inner Galaxy
}

\author{
Sofia Ventura ${ }^{a, *}$ \\ ${ }^{a}$ Università di Siena and INFN Pisa, \\ I-53100 Siena, Italy \\ E-mail: sofia.ventura@pi.infn.it
}

The origin of the high-energy gamma-ray emission from the Milky Way center is still unclear and debated because of the impact of systematics afflicting the measurements from current experiments. Several theories and phenomenological models attempt to explain the intricate panorama. The presence of a PeVatron in the Central Molecular Zone or in its vicinity, the contribution of the hard-component of the diffuse gamma-ray emission, and dark matter annihilation scenario are among the most promising mechanisms for describing the observed excess. The development of increasingly precise models able to reproduce the measured gamma-ray emission is the challenge for the scientific community in view of the next generation telescopes.

A detailed treatment of phenomenological models for the dubbed Cosmic Rays Sea (CR-sea) characterized by different configurations is scrutinized in comparison with the observed spectrum in the inner Galaxy, using DRAGON and GAMMASKY codes.

$37^{\text {th }}$ International Cosmic Ray Conference (ICRC 2021)

July 12 th - 23rd, 2021

Online - Berlin, Germany

\footnotetext{
${ }^{*}$ Presenter
} 


\section{Introduction}

Our Own Galaxy is known to be a strong source of diffuse $\gamma$-ray emission [1,2]. First EGRET instrument measured its flux over the full sky ( $E=0.03 \div 10 \mathrm{GeV}$, [3]). The next generation telescope Fermi-LAT - onboard the NASA GLAST satellite - measured with better accuracy the diffuse emission from the whole Galactic Plane (GP) up to than $100 \mathrm{GeV}$ [4].

The most significant component of the diffuse emission is due to the decay of neutral pions $\left(\pi^{0}\right)$, as consequence of the collisions of the Galactic Cosmic Rays (CRs) hadronic component with the gaseous structure of the Interstellar Medium (ISM, [5, 6]): the column density of the interstellar matter is traced by the pionic flavour of the Galactic diffuse $\gamma$-ray emission. The leptonic component of CRs contributes to this emission via Inverse Compton (IC) scattering and bremsstrahlung, but with different spectral shape and spatial distribution allowing to disentangle its effect to the measured flux.

The Imaging Cherenkov Atmospheric Technique (IACT) telescopes observe the Very High Energy $(E>100 \mathrm{GeV}, \mathrm{VHE}) \gamma$-rays from the Earth ground [7, 8]: the restricted field-of-view (FOV) of these telescopes allows to study the diffuse emission in specific regions of interest, but with much higher angular resolution, and increasing the reachable energy range with respect to orbital observatories - like Fermi-LAT characterized by a lower energy sensitivity, but a broader FOV.

Understanding and modeling the $\gamma$-ray diffuse emission, especially at higher energies, is fundamental in the High Energy (HE) Astrophysics community: the capability to describe and reproduce the CR transport within Our Galaxy, and the subsequently interactions with the interstellar gas - locked in complex structures - gives the opportunity to estimate the expected emission in each part of the Milky Way. The reproducibility of the so-called large-scale CR-sea is an important aspect in the current, and especially, next generation telescopes since it has a key-role in the definition of the background model, a fundamental ingredient in the analysis-chain of measured data.

\section{The Central Molecular Zone}

The Galactic Center (GC) of the Milky Way is the perfect laboratory for studying phenomena and physical processes that may be occurring in many other galactic nuclei. The observations show the evidence for a central massive black hole - the compact radio source Sgr A ${ }^{\star}$ - and a dense and luminous star cluster, as well as several components of neutral, ionized, and hot gas [9]. The inner part of the GC is represented by the so-called Central Molecular Zone (CMZ) which represents one of the densest environment of the Galaxy.

The first measurements of energy spectrum and spatial distribution of the diffuse $\gamma$-ray emission from the GC region was provided by the H.E.S.S. collaboration in 2006 [10]. That emission was found tracing the column density of the interstellar gas (probed by the related CO and CS molecular emission lines) and extends over about 2 degrees in Galactic longitude along the GP: namely the Central Galactic Ridge region. This is the first evidence of an harder spectrum than that measured at the Earth position ${ }^{1}$.

\footnotetext{
${ }^{1}$ It was expected to be the same in the whole Galaxy.
} 
In 2016 the H.E.S.S. collaboration released new data with much higher statistics ( 250 hours of exposure) allowing to extend the explored energy range, and to perform a better study of the morphology of the emission [11, 12]. This flux was supposed to be originated by the interaction of high energy CR hadrons (mostly protons) with the dense gas in the GC region giving rise to $\pi^{0} \mathrm{~s}$, which rapidly decay into $\gamma$-rays. Assuming that the emission is originated by proton-proton scattering, this implies the presence of a population of primary CRs in that region extending up to energies close to $\sim 1 \mathrm{PeV}$ with a power-law (PL) spectrum with index close to $\sim 2.4$ due to the slowly increasing behaviour of the $p p$-scattering cross section with energy (see [13]). The presence of strong magnetic fields ( $\sim 100 \mu \mathrm{G}$ [14]), and intense infrared (IR) radiation field produces relevant synchrotron and IC losses above the TeVs: the CR leptonic component is not expected to contribute significantly to the observed emission.

The $\gamma$-ray diffuse emission traces the whole molecular gas complex in the GC (the CMZ), which approximates a cylindrical region of $\sim 250 \mathrm{pc}$ radius from the center. The inner part of the CMZ (the Galactic Ridge, $\left.|l|<1^{\circ},|b|<0.3^{\circ}\right)^{2}$, approximately corresponds to a symmetric cylinder of $\sim 30 \mathrm{pc}$ thickness and $\simeq 170 \mathrm{pc}$ long, around Sgr $\mathrm{A}^{\star}$. The H.E.S.S. collaboration [12] measured a uniform spectral index $\Gamma_{\mathrm{HESS} 18}=-2.28 \pm 0.03_{\text {stat }} \pm 0.2_{\text {syst }}$ up to $45 \mathrm{TeV}$, suggesting that a single population of particles fills the whole CMZ.

The H.E.S.S. results have raised a wide interest in the Astroparticle community as they may provide the first evidence of a PeVatron in the Milky Way.

\section{Background Models for the CMZ}

A nowadays important research field is represented by the efforts of the scientific community in modelling the large-scale $\gamma$-ray background. In figure 1 are reported four views of the expected emission from the CMZ at $10 \mathrm{GeV}$. Starting from the top left, Gamma Model, Gamma Model without hardening, Base Model, and Conventional description are shown. In this work is briefly described the characteristics of such models.

The so-called Gamma Model is obtained as the solution of transport equation - solved by DRAGON code [15] - and integration along the line-of-sight of the CR density propagated in the whole Galaxy (using GAMMASKY code $[16,17]$ ). This model is featured by the radial dependence of both the scaling of the CR diffusion coefficient with rigidity, and the advection velocity (for major details see [18]). This feature is able to reproduce the hardening of the CR spectral index observed in the inner Galaxy (see [19, 20]). Moreover, an additional hardening is present at $\sim 300 \mathrm{GeV} / \mathrm{n}$ as measured by PAMELA [21], AMS-02 [22] and CREAM [23]. This second feature reproduces the observed MILAGRO anomaly at $15 \mathrm{TeV}$ [24]. In the discussion is also proposed a view of the background model without the additional hardening at $300 \mathrm{GeV} / \mathrm{n}$ (Gamma Model without hardening).

The third model proposed is the so-called Base Model: the diffusion coefficient is considered constant in the whole Galaxy, and corresponding to the value observed at the Earth position $(\Gamma \sim 2.6 \div 2.7[25])$. Even in this case, the hardening at $300 \mathrm{GeV} / \mathrm{n}$ is considered. The last representation is the Conventional description of the CR diffusion.

\footnotetext{
${ }^{2} 1^{\circ}$ corresponds to $\sim 150 \mathrm{pc}$ at the GC distance $(\sim 8.5 \mathrm{kpc})$.
} 

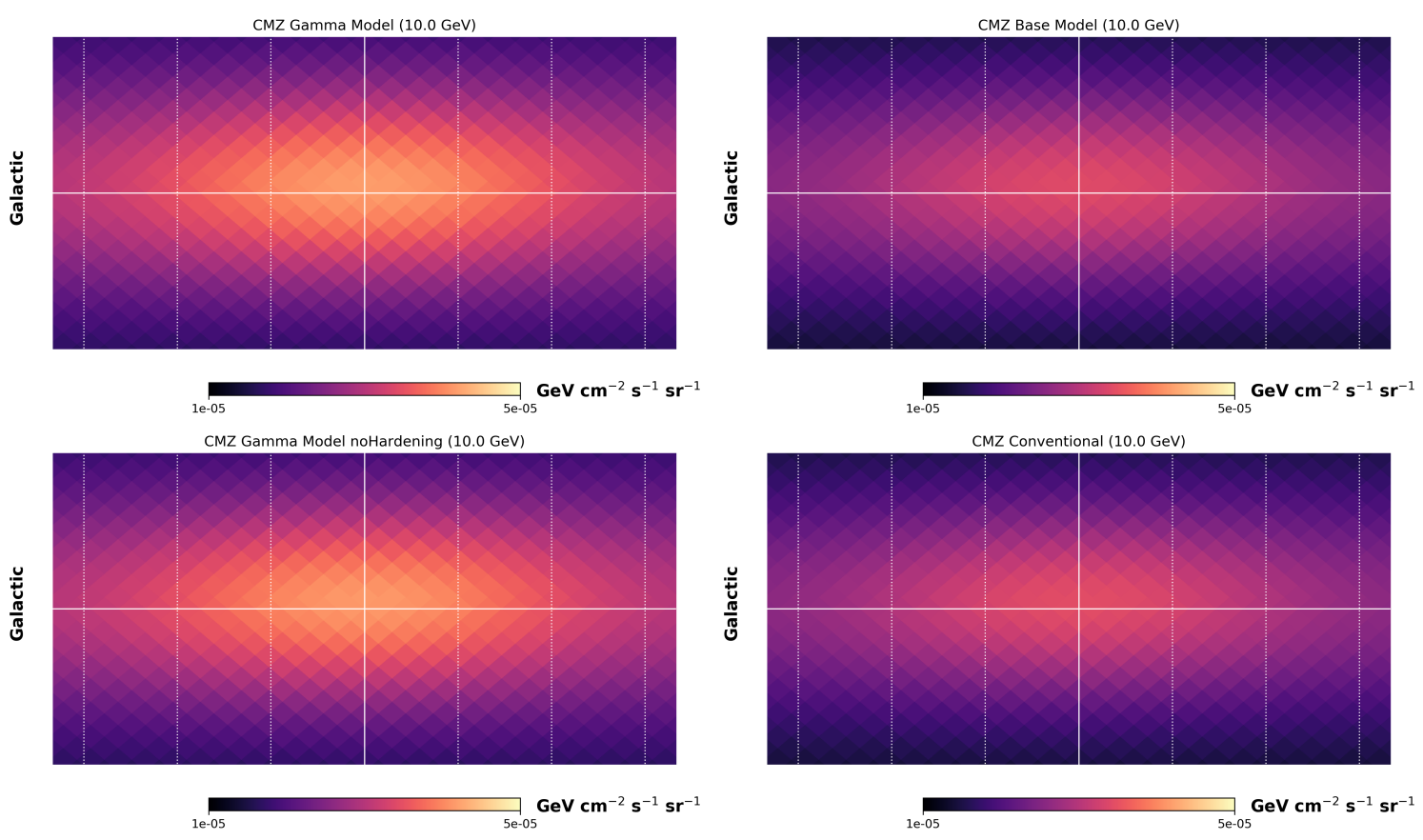

Figure 1: View of the $\gamma$-ray expected emission as output of Gamma Model, Gamma Model without hardening, Base Model and Conventional description. The representations are obtained at $10 \mathrm{GeV}$, and in a region corresponding to the Galactic Ridge $\left(|l|<1^{\circ},|b|<0.3^{\circ}\right)$.

The obtained CR distributions (with DRAGON) are included as inputs of GAMMASKY code. For modeling the gas structure and distribution of the inner ring of Our Galaxy, an analytical model is used as described in [26]. This model represents a source of systematics since the corresponding distribution of the gaseous content is smooth: there is not a detailed description of the clouds and complex structures filling the CMZ.

Since the observations of the inner ring of our Galaxy are affected by large uncertainties and systematics, the reported hypothesis represents an extension at the GC position of the trend tuned on local data, and an extrapolation at higher energies. A development of Gamma Model is ongoing, tuned on recent observations and featured by updated maps of the gas distribution in the Galaxy. The next released large-scale models of $\gamma$-ray emission from the whole Milky Way will be released with the use of DRAGON2 [27] and HERMES codes ${ }^{3}$. These tools are usable to the scientific community.

\section{Results}

The models described above are compared with the observed data by currently operating gamma-ray observatories. In particular, a detailed analysis of $\sim 10$ years of Pass8 [28] Fermi-LAT data has been performed with the latest released IRFs (P8R3_CLEAN_V2), isotropic background (iso_P8R3_CLEAN_V2), and 4FGL catalog [29].

In figure 2 left is shown the energy spectrum from $1 \mathrm{GeV}$ to more than $50 \mathrm{TeV}$ of the $\gamma$-ray emission from the inner part of the CMZ, the Galactic Ridge (for more details see [12]).

\footnotetext{
3https://github.com/cosmicrays/
} 
In this work the analyzed Fermi-LAT data is compared with H.E.S.S. [12], MAGIC [30] and VERITAS [31] measurements. Moreover the expected diffuse $\gamma$-ray emission of the four phenomenological models described in the previous section is shown.

At the Fermi-LAT energies the models are able to reproduce the observed excess in the Galactic Ridge, while at higher energies - essentially at that energies reachable by Cherenkov Telescopes the four energy spectra reproduce a different behaviour of the modelled $\gamma$-ray diffuse emission. In particular the measured emission is reproduced by the Gamma Model, but not properly by the other three models.

Instead, in figure 2 right, the measured Fermi-LAT and H.E.S.S. data are compared with the expected $\gamma$-ray emission computed with Gamma Model. The blue band represents the uncertainty in the normalization of the spectrum of the diffuse emission. Indeed in [26] for estimating the gas mass of the inner ring of Our Galaxy, has been considered the so-called $X_{\mathrm{CO}}$ factor [32] equals to $\sim(2 \div 4) \times 10^{19} \mathrm{~cm}^{-2} \mathrm{~K}^{-1} \mathrm{~km}^{-1} \mathrm{~s}$ with an uncertainty of order 2 . This causes a corresponding uncertainty in the prediction of the model used to compute the expected $\gamma$-ray emission from the region.
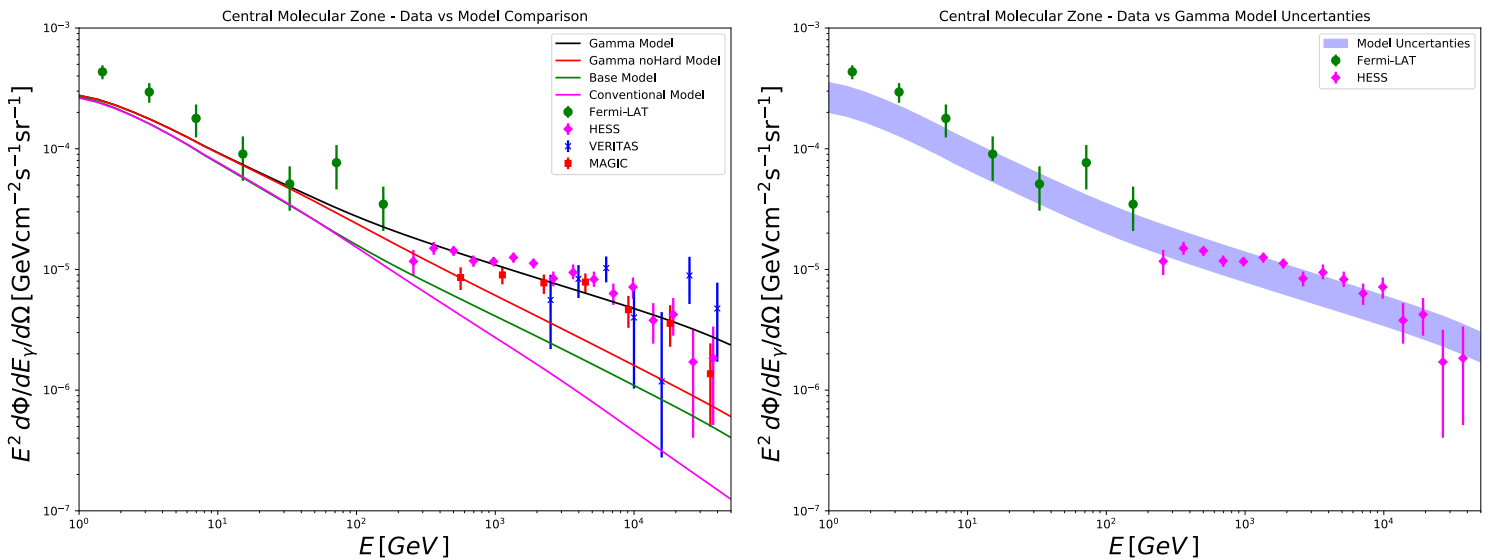

Figure 2: Left: Compared energy spectra of $\gamma$-ray diffuse emission from the Galactic Ridge with Fermi-LAT, H.E.S.S. [12], MAGIC [30] and VERITAS [31] data. Right: Gamma Model energy spectrum compared with Fermi-LAT and H.E.S.S. data in the Galactic Ridge region. The blue band represents the systematic error due to the uncertainty in the estimation of the $X_{\mathrm{CO}}$ factor at the GC position.

\section{Discussion and Conclusions}

In the H.E.S.S. Collaboration papers $[11,12]$ the observed $\gamma$-ray excess has been interpreted in terms of the so-called PeVatron scenario. The inferred CR density is compatible with CR escaping from a steady-state source that continuously injects CRs in the region. The presence of a PeVatron is associated with the Super Massive Black Hole (SMBH) Sgr A* in the center of the Milky Way, or with a hidden accelerator located in its vicinity, for instance associated with a compact Stellar Wind Cluster [33, 34].

In this paper instead, the observed flux from the Galactic Ridge has been interpreted in terms of the hard component of the diffuse $\gamma$-ray emission featured by the radial dependence of the diffusion 
coefficient, and an hardening at $300 \mathrm{GeV} / \mathrm{n}$. As mentioned above, this hypothesis represents an extension at the GC position of the behaviour observed between $8 \mathrm{kpc}$ and $3 \mathrm{kpc}$, and tuned on local data. Moreover, the extrapolation at higher energies - the energies reachable by IACTs - is strongly dependent on the CR transport parameters variation (figure 2 left).

Both PeVatron scenario and hard-diffusion scenario are able to reproduce the observed spectrum in the innermost part of Our Own Galaxy: with the currently operating $\gamma$-ray observatories it is not possible to know with sufficient accuracy the origin and the nature of the observed $\gamma$-ray excess, and to reach definitive conclusions.

The opportunity to clarify the intricate panorama featuring the center of the Milky Way is given by the observation of different regions farther from the GC. For instance, the recently discovered HESS J1741-302 [35] may represent an ideal target for testing the impact of different scenarios (for more details see [36]). Another interesting region is represented by Bania Clump, a complex, dense and giant molecular cloud located at $\sim 500 \div 800 \mathrm{pc}$ away from the GC (see [37]). Indeed these kind of objects represent ideal targets for CR hadrons interaction and subsequently $\gamma$-rays production. Whether the presence of potential PeVatron sources is sufficiently far way from selected peculiar dense clouds, the well-know $1 / r$ CR radial profile is not able to shine these regions, but the level of the $\gamma$-ray background emission featured by the hardening, described above, may be high enough to illuminate dense clouds. In this context, an accurate 3D description of the morphology and dynamics of the clouds filling the inner part of Our Galaxy is fundamental and required.

The opportunity to disentangle among the several suggested scenarios will be given by the upcoming Cherenkov Telescope Array (CTA) thanks to the increased sensitivity and angular resolution. At that point, the nowadays dark regions - too faint to be detected by currently operating $\gamma$-ray observatories - may be bright enough to be detected by CTA, and shed light on the origin of this peculiar emission. Moreover the detection of a cut-off in the observed energy spectrum will give the definitive interpretation on the nature and origin of the emission independently by models [38].

\section{References}

[1] C.E. Fichtel, R.C. Hartman, D.A. Kniffen, D.J. Thompson, H. Ogelman, M.E. Ozel et al., High-energy gamma-ray results from the second small astronomy satellite, ApJ 198 (1975) 163.

[2] W.L. Kraushaar, G.W. Clark, G.P. Garmire, R. Borken, P. Higbie, V. Leong et al., High-Energy Cosmic Gamma-Ray Observations from the OSO-3 Satellite, ApJ 177 (1972) 341.

[3] S.D. Hunter, D.L. Bertsch, J.R. Catelli, T.M. Dame, S.W. Digel, B.L. Dingus et al., EGRET Observations of the Diffuse Gamma-Ray Emission from the Galactic Plane, ApJ 481 (1997) 205.

[4] M. Ackermann, M. Ajello, W.B. Atwood, L. Baldini, J. Ballet, G. Barbiellini et al., Fermi-LAT Observations of the Diffuse $\gamma$-Ray Emission: Implications for Cosmic Rays and the Interstellar Medium, ApJ 750 (2012) 3 [1202 . 4039]. 
[5] F.W. Stecker, The Cosmic $\gamma$-Ray Spectrum from Secondary Particle Production in Cosmic-Ray Interactions, Ap\&SS 6 (1970) 377.

[6] C.D. Dermer, Secondary production of neutral pi-mesons and the diffuse galactic gamma radiation, A\&A 157 (1986) 223.

[7] F.A. Aharonian, W. Hofmann, A.K. Konopelko and H.J. Völk, The potential of ground based arrays of imaging atmospheric Cherenkov telescopes. I. Determination of shower parameters, Astroparticle Physics 6 (1997) 343.

[8] F.A. Aharonian, W. Hofmann, A.K. Konopelko and H.J. Völk, The potential of the ground based arrays of imaging atmospheric Cherenkov telescopes. II. Gamma ray flux sensitivities, Astroparticle Physics 6 (1997) 369.

[9] R. Genzel, F. Eisenhauer and S. Gillessen, The Galactic Center massive black hole and nuclear star cluster, Reviews of Modern Physics 82 (2010) 3121 [1006. 0064].

[10] F. Aharonian, A.G. Akhperjanian, A.R. Bazer-Bachi, M. Beilicke, W. Benbow, D. Berge et al., Discovery of very-high-energy $\gamma$-rays from the Galactic Centre ridge, Nature 439 (2006) 695 [astro-ph/0603021].

[11] HESS Collaboration, A. Abramowski, F. Aharonian, F.A. Benkhali, A.G. Akhperjanian, E.O. Angüner et al., Acceleration of petaelectronvolt protons in the Galactic Centre, Nature 531 (2016) 476 [1603.07730].

[12] H. E. S. S. Collaboration, H. Abdalla, A. Abramowski, F. Aharonian, F. Ait Benkhali, A.G. Akhperjanian et al., Characterising the VHE diffuse emission in the central 200 parsecs of our Galaxy with H.E.S.S., A\&A 612 (2018) A9 [1706. 04535].

[13] Particle Data Group collaboration, Review of Particle Physics, Chin. Phys. C40 (2016) 100001.

[14] R.M. Crocker, D.I. Jones, F. Melia, J. Ott and R.J. Protheroe, A lower limit of 50 microgauss for the magnetic field near the Galactic Centre, Nature 463 (2010) 65 [1001. 1275].

[15] C. Evoli, D. Gaggero, D. Grasso and L. Maccione, Cosmic ray nuclei, antiprotons and gamma rays in the galaxy: a new diffusion model, J. Cosmology Astropart. Phys. 10 (2008) 018 [0807 . 4730].

[16] G. Di Bernardo, C. Evoli, D. Gaggero, D. Grasso and L. Maccione, Cosmic ray electrons, positrons and the synchrotron emission of the Galaxy: consistent analysis and implications, .

[17] C. Evoli, D. Gaggero, D. Grasso and L. Maccione, Common Solution to the Cosmic Ray Anisotropy and Gradient Problems, Physical Review Letters 108 (2012) 211102 [1203.0570].

[18] D. Gaggero, M. Taoso, A. Urbano, M. Valli and P. Ullio, Towards a realistic astrophysical interpretation of the gamma-ray Galactic center excess, J. Cosmology Astropart. Phys. 12 (2015) 056 [1507.06129]. 
[19] F. Acero, M. Ackermann, M. Ajello, A. Albert, L. Baldini, J. Ballet et al., Development of the Model of Galactic Interstellar Emission for Standard Point-source Analysis of Fermi Large Area Telescope Data, ApJS 223 (2016) 26 [1602 . 07246].

[20] R. Yang, F. Aharonian and C. Evoli, Radial distribution of the diffuse $\gamma$-ray emissivity in the Galactic disk, Phys. Rev. D 93 (2016) 123007 [1602 . 04710].

[21] O. Adriani, G.C. Barbarino, G.A. Bazilevskaya, R. Bellotti, M. Boezio, E.A. Bogomolov et al., PAMELA Measurements of Cosmic-Ray Proton and Helium Spectra, Science 332 (2011) 69 [1103.4055].

[22] M. Aguilar, D. Aisa, B. Alpat, A. Alvino, G. Ambrosi, K. Andeen et al., Precision Measurement of the Proton Flux in Primary Cosmic Rays from Rigidity 1 GV to 1.8 TV with the Alpha Magnetic Spectrometer on the International Space Station, Physical Review Letters 114 (2015) 171103.

[23] H.S. Ahn, P. Allison, M.G. Bagliesi, J.J. Beatty, G. Bigongiari, J.T. Childers et al., Discrepant Hardening Observed in Cosmic-ray Elemental Spectra, ApJ 714 (2010) L89 [1004.1123].

[24] D. Gaggero, D. Grasso, A. Marinelli, A. Urbano and M. Valli, The Gamma-Ray and Neutrino Sky: A Consistent Picture of Fermi-LAT, Milagro, and IceCube Results, ApJ 815 (2015) L25 [1504 . 00227].

[25] M. Tanabashi, K. Hagiwara, K. Hikasa, K. Nakamura, Y. Sumino, F. Takahashi et al., Review of Particle Physics*, Phys. Rev. D 98 (2018) 030001.

[26] K. Ferrière, W. Gillard and P. Jean, Spatial distribution of interstellar gas in the innermost 3 kpc of our galaxy, A\&A 467 (2007) 611 [astro-ph/0702532].

[27] C. Evoli, D. Gaggero, A. Vittino, G. Di Bernardo, M. Di Mauro, A. Ligorini et al., Cosmic-ray propagation with DRAGON2: I. numerical solver and astrophysical ingredients, J. Cosmology Astropart. Phys. 2 (2017) 015 [1607. 07886].

[28] P. Bruel, T.H. Burnett, S.W. Digel, G. Johannesson, N. Omodei and M. Wood, Fermi-lat improved pass 8 event selection, 2018.

[29] S. Abdollahi, F. Acero, M. Ackermann, M. Ajello, W.B. Atwood, M. Axelsson et al., Fermi large area telescope fourth source catalog, The Astrophysical Journal Supplement Series 247 (2020) 33.

[30] MAGIC Collaboration, V.A. Acciari, S. Ansoldi, L.A. Antonelli, A. Arbet Engels, D. Baack et al., MAGIC observations of the diffuse $\gamma$-ray emission in the vicinity of the Galactic center, A\&A 642 (2020) A190 [2006.00623].

[31] C.B. Adams, W. Benbow, A. Brill, R. Brose, M. Buchovecky, M. Capasso et al., VERITAS Observations of the Galactic Center Region at Multi-TeV Gamma-Ray Energies, ApJ 913 (2021) 115 [2104.12735]. 
[32] R.L. Dickman, A survey of carbon monoxide emission in dark clouds, ApJ 202 (1975) 50.

[33] F. Aharonian, R. Yang and E. de Oña Wilhelmi, Massive stars as major factories of Galactic cosmic rays, Nature Astronomy 3 (2019) 561 [1804 . 02331].

[34] G. Morlino, P. Blasi, E. Peretti and P. Cristofari, Particle acceleration in winds of star clusters, MNRAS 504 (2021) 6096 [2102 . 09217].

[35] H. E. S. S. Collaboration, H. Abdalla, A. Abramowski, F. Aharonian, F. Ait Benkhali, E.O. Angüner et al., HESS J1741-302: a hidden accelerator in the Galactic plane, A\&A 612 (2018) A13 [1711.01350].

[36] S. Ventura, D. Grasso and A. Marinelli, Shedding (Gamma) Light on the Cosmic Ray Population in the Galactic Center Region, in 36th International Cosmic Ray Conference (ICRC2019), vol. 36 of International Cosmic Ray Conference, p. 816, July, 2019.

[37] S. Ventura, Cosmic Rays Shining in the Galactic Center: A Study of Gamma-Ray Diffuse Emission of the Central Molecular Zone, Master Thesis (ETD UNIPI) (2018) [etd-03272018-145514].

[38] S. Celli, F. Aharonian and S. Gabici, Spectral Signatures of PeVatrons, ApJ 903 (2020) 61 [2009.05999]. 